\title{
ORBITS IN UNIMODULAR HERMITIAN LATTICES
}

\author{
DONALD G. JAMES
}

\begin{abstract}
Let $L$ be a unimodular indefinite hermitian lattice over the integers 0 of an algebraic number field, and $N(L, c)$ the number of primitive representations of $c \in 0$ by $L$ that are inequalivant modulo the action of the integral special unitary group $S U(L)$ on $L$. The value of $N(L, c)$ is determined from the local representations via a product formula.
\end{abstract}

\section{INTRODUCTION}

Let $F$ be an algebraic number field and $K$ a quadratic extension of $F$. Let $V$ be an indefinite hermitian space over $K$ of finite dimension $n \geq 3$ and $f: V \times V \rightarrow K$ the associated nondegenerate hermitian form on $V$ with respect to the nontrivial automorphism * of $K$ over $F$ where $f(a x, b y)=$ $a^{*} f(x, y) b$. Assume $V$ supports a unimodular lattice $L$ (in the sense of O'Meara [O'M, 82G] for quadratic spaces). Denote by $U(V)$ the unitary group of $V$ and by $U(L)$ the subgroup of isometries in $U(V)$ that leave $L$ invariant. We study the orbits of primitive elements in $L$ under the action of the special unitary group $S U(L)=U(L) \cap S L(V)$. The problem is first solved locally; the global result is then obtained by applying the strong approximation theorem of Shimura [S, 5.12]. The analogous problem for quadratic lattices was considered in [J4]; earlier work on this integral version of Witt's theorem can be found in $[\mathrm{J} 1, \mathrm{~J} 2, \mathrm{~N}$ and $\mathrm{W}]$. Kilhefner $[\mathrm{K}]$ gives hermitian analogues for the results in [J1].

Let $\Omega_{F}$ be the set of all nontrivial prime spots on $F$ and $S$ the set of all finite prime spots. Denote by $\mathfrak{o}=\mathfrak{o}_{F}$ the associated Dedekind ring of algebraic integers. Let $\mathfrak{O}_{K}$ be the integral closure of $\mathfrak{o}_{F}$ in $K$. Although $L$ is an $\mathfrak{O}_{K^{-}}$ module, most of the calculations are done locally at the dyadic primes in $S$ that ramify in $K$. The localization procedure followed here is essentially that first studied by Shimura [S] (see also [G]). Let $\mathfrak{p}$ be a prime spot of $F$ and $F_{\mathfrak{p}}$ the corresponding local field. Put $K_{\mathfrak{p}}=K \otimes_{F} F_{\mathfrak{p}}$ and $V_{\mathfrak{p}}=V \otimes_{F} F_{\mathfrak{p}}$. Making the standard identifications, we have $K \subset K_{\mathfrak{p}}, F_{\mathfrak{p}} \subset K_{\mathfrak{p}}$ and $V \subset V_{\mathfrak{p}}$. The hermitian form $f$ on $V$ extends naturally to a hermitian from on $V_{\mathfrak{p}}$. For

Received by the editors June 18, 1990.

1980 Mathematics Subject Classification (1985 Revision). Primary 11E39; Secondary 20G25, $20 \mathrm{G} 30$.

Key words and phrases. Unimodular hermitian forms, algebraic integers, integral representations, unitary group, orbits, local fields, cyclotomic fields.

This research was partially supported by the National Science Foundation. 
each $\mathfrak{p} \in S$, let $\mathfrak{o}_{\mathfrak{p}}$ be the topological closure of $\mathfrak{o}$ in $F_{\mathfrak{p}}$, and $\mathfrak{O}_{\mathfrak{p}}$ the integral closure of $\mathfrak{o}_{\mathfrak{p}}$ in $K_{\mathfrak{p}}$. Put $L_{\mathfrak{p}}=\mathfrak{O}_{\mathfrak{p}} L \subset V_{\mathfrak{p}}$. Then $L_{\mathfrak{p}}$ is locally unimodular so that $f\left(L_{\mathfrak{p}}, L_{\mathfrak{p}}\right)=\mathfrak{O}_{\mathfrak{p}}$ and $L_{\mathfrak{p}}$ has a basis with $\operatorname{det} f\left(x_{i}, x_{j}\right)$ a unit in $\mathfrak{O}_{\mathfrak{p}}$. Note when $\mathfrak{p}$ splits in $K$ that $K_{\mathfrak{p}}=F_{\mathfrak{p}} \times F_{\mathfrak{p}}, \mathfrak{O}_{\mathfrak{p}}=\mathfrak{o}_{\mathfrak{p}} \times \mathfrak{o}_{\mathfrak{p}}$ and the involution * on $K$ becomes $(a, b)^{*}=(b, a)$ on $K_{\mathfrak{p}}$.

An element $x \in L$ is called primitive if $f(x, L)=\mathfrak{O}_{K}$. We say $L$ represents $c \in \mathfrak{o}$ if there exists a primitive $x \in L$ with $f(x)=f(x, x)=c$ (all our representations are understood to be primitive). Put

$$
L(c)=\left\{x \in L \mid f(x)=c \text { and } f(x, L)=\mathfrak{O}_{K}\right\}
$$

and let $N(L, c)$ be the number of orbits in $L(c)$ under the action of $S U(L)$, that is, the number of primitive representation of $c$ modulo the action of $S U(L)$. Similarly, denote by $N\left(L_{\mathfrak{p}}, c\right)$ the corresponding number of local primitive representations of $c \in \mathfrak{o}_{\mathfrak{p}}$ modulo the action of $S U\left(L_{\mathfrak{p}}\right)$. These representation numbers, $N(L, c)$ and $N\left(L_{\mathfrak{p}}, c\right)$, will be seen in $\S \S 4$ and 5 to be finite. Representations of $c$ by $x$ and $y$ are called (locally or globally) equivalent when $x$ and $y$ lie in the same orbit.

Theorem 1.1. Let $L$ be a unimodular lattice on an indefinite hermitian space $V$ with dimension $n \geq 3$. Let $c$ be a nonzero element in o represented by $L$ and assume $V \perp\langle-c\rangle$ has local Witt index at least two for some $\mathfrak{q} \notin S$. Then

$$
N(L, c)=\prod_{\mathfrak{p}} N\left(L_{\mathfrak{p}}, c\right)
$$

where the product is taken over all dyadic primes $\mathfrak{p} \in S$ that ramify in $K$.

Note that the Witt index condition on $V \perp\langle-c\rangle$ is always satisfied when there exists a spot $\mathfrak{q} \notin S$ which splits in $K$. For example, the condition is satisfied if $K \subset \mathbf{R}$, the real field. The values of $N(L, c)$ will be explicitly calculated in some special cases, including $F=\mathbf{Q}$ (see Theorem 6.1), or $K$ a cyclotomic field (see $\S 7$ ). Since the structure of local hermitian lattices is simpler than that of local quadratic lattices, the final results here will be more general than those in [J4].

\section{Global ORBITS}

The following result reduces the question of global equivalence of representations of the corresponding local problems.

Theorem 2.1. Let $L$ be a unimodular lattice on a hermitian space $V$ with dimension $n \geq 3$. Let $c$ be a nonzero element in $o_{F}$ and assume $V \perp\langle-c\rangle$ has local Witt index at least two for some $\mathfrak{q} \notin S$. Let $x, y \in L(c)$ be locally equivalent at each $\mathfrak{p} \in S$. Then there exists $\varphi \in S U(L)$ such that $\varphi(x)=y$.

Proof. By hypothesis, there exist local $\varphi_{\mathfrak{p}} \in S U\left(L_{\mathfrak{p}}\right)$ such that $\varphi_{\mathfrak{p}}(x)=y$ for each $\mathfrak{p} \in S$. By Witt's theorem, there exists $\theta \in U(V)$ such that $\theta(x)=y$. Let $\operatorname{det} \theta=\eta$. Then the norm $N_{K / F}(\eta)=1$. Take $r \in V$ with $f(r) \neq 0$ and $f(r, x)=0$. Since the quasi-symmetry

$$
\Psi(r): z \mapsto z-(1-\eta) f(r, z) f(r)^{-1} r, \quad z \in V,
$$

fixes $x$ and $\operatorname{det} \theta \Psi(r)^{-1}=1$, we may assume $\theta \in S U(V)$. Then $\theta_{\mathfrak{p}}^{-1} \varphi_{\mathfrak{p}}(x)=x$ for each $\mathfrak{p} \in S$. Let $V=K x \perp W$. Then $\theta_{\mathfrak{p}}^{-1} \varphi_{\mathfrak{p}} \in S U\left(W_{\mathfrak{p}}\right), \operatorname{dim} W \geq 2$ and 
$W_{\mathfrak{q}}$ is indefinite for some $\mathfrak{q} \notin S$. By the strong approximation theorem on $S U(W)$ [S, 5.12] (and modifying the norm notation \|\|$_{\mathfrak{p}}$ from [O'M, 101]), there exists $\psi \in S U(W)$ such that $\left\|\psi-\theta_{\mathfrak{p}}^{-1} \varphi_{\mathfrak{p}}\right\|_{\mathfrak{p}}<\varepsilon$ for the finite number of $\mathfrak{p}$ in $S$ where $\|\theta\|_{\mathfrak{p}} \neq 1$, and $\|\psi\|_{\mathfrak{p}}=1$ for all remaining $\mathfrak{p}$ in $S$. Extend $\psi$ by the identity to $S U(V)$ and put $\varphi=\theta \psi \in S U(V)$. Then $\varphi(x)=y$ and $\|\varphi\|_{\mathfrak{p}}=1$ for all $\mathfrak{p} \in S$, provided $\varepsilon$ was chosen sufficiently small initially. Hence $\varphi \in S U(L)$.

The proof of Theorem 1.1 is similar to that for the analogous result in quadratic spaces, namely, Theorem 2.3 in [J4]; it will be given after some local results have been developed.

\section{LOCAL ORBITS}

The norm and trace mappings from $K_{\mathfrak{p}}$ to $F_{\mathfrak{p}}$ are denoted by $N_{\mathfrak{p}}$ and $T_{\mathfrak{p}}$, respectively. For each $\mathfrak{p} \in S$, denote by $L_{\mathfrak{p}}^{\prime}$ the sublattice of $L_{\mathfrak{p}}$ generated by the $x \in L_{\mathfrak{p}}$ with $f(x) \in 2 \mathfrak{o}_{\mathfrak{p}}$. Trivially, $L_{\mathfrak{p}}^{\prime}=L_{\mathfrak{p}}$ for any nondyadic $\mathfrak{p}$. Now suppose $\mathfrak{p}$ splits in $K$. Then, since the involution on $\mathfrak{O}_{\mathfrak{p}}=\mathfrak{o}_{\mathfrak{p}} \times \mathfrak{o}_{\mathfrak{p}}$ is given by $(a, b)^{*}=(b, a)$, for any $x \in L_{\mathfrak{p}}$ we have $f((1,0) x)=N_{\mathfrak{p}}(1,0) f(x)=0$. Hence $(1,0) x \in L_{\mathfrak{p}}^{\prime}$ and $x=(1,1) x$ is in $L_{\mathfrak{p}}^{\prime}$, therefore $L_{\mathfrak{p}}^{\prime}=L_{\mathfrak{p}}$. When $\mathfrak{p} \in S$ is not split in $K$, let $\mathfrak{P}$ denote the unique maximal ideal in $\mathfrak{O}_{\mathfrak{p}}$. Assume $x \in L_{\mathfrak{p}}$ is primitive. Then $f\left(x, L_{\mathfrak{p}}^{\prime}\right)=\mathfrak{P}^{m}$ for some $m \geq 0$. Here $m=m_{\mathfrak{p}}(x)$ is called the degree. Moreover, $m=0$ when $\mathfrak{p}$ is nondyadic. By convention we set $m=0$ when $\mathfrak{p}$ is split. Call $x$ characteristic when $m_{\mathfrak{p}}(x) \geq 1$ (p must then be dyadic).

Theorem 3.1. Assume $L_{\mathfrak{p}}$ is unimodular with $n=\operatorname{rank} L_{\mathfrak{p}} \geq 3$ and $x, y$ are primitive in $L_{\mathfrak{p}}$. Then there exists $\varphi \in S U\left(L_{\mathfrak{p}}\right)$ such that $\varphi(x)=y$ if and only if

(i) $f(x)=f(y)$,

(ii) $m_{\mathfrak{p}}(x)=m_{\mathfrak{p}}(y)=m$,

(iii) $x-y \in \mathfrak{P}^{m} L_{\mathfrak{p}}^{\prime}$.

This theorem provides the information needed to calculate $N\left(L_{\mathfrak{p}}, c\right)$. To prove the theorem we must first determine the structure of $L_{\mathfrak{p}}^{\prime}$ at the dyadic primes and find generators for $S U\left(L_{\mathfrak{p}}\right)$. Both of those problems have already been investigated in [J3].

When $\mathfrak{p}$ does not split in $K$, let $K_{\mathfrak{p}}=F_{\mathfrak{p}}(\zeta)$ where $\zeta^{2} \in \mathfrak{o}_{\mathfrak{p}}$ and $\zeta^{*}=-\zeta$. Fix a prime $\pi$ in $K_{\mathfrak{p}}$ and $p$ in $F_{\mathfrak{p}}$ and let $e=\operatorname{ord}_{p} 2$. In the nondyadic case $\mathfrak{O}_{\mathfrak{p}}$ is generated over $\mathfrak{o}_{\mathfrak{p}}$ by 1 and $\zeta$ provided we choose $\zeta$ to be a prime or a unit according as the extension is ramified or not. If $\mathfrak{p}$ is dyadic, there are the following three possible types of extension of $K_{\mathfrak{p}}$ over $F_{\mathrm{p}}$ (see [Jc] or [O'M, 63.2 and 63.3] for more details).

(i) $K_{\mathfrak{p}}$ is an unramified extension of $F_{\mathfrak{p}}$. Then $\zeta^{2}=1+4 \delta$ with $\delta$ a unit in $\mathfrak{o}_{\mathfrak{p}}$, and $\mathfrak{O}_{\mathfrak{p}}$ consists of the elements $(\alpha+\zeta \beta) / 2$ with $\alpha, \beta \in \mathfrak{o}_{\mathfrak{p}}$ and $\alpha \equiv \beta \bmod 2$. Here $2 \mathfrak{O}_{\mathfrak{p}}=\mathfrak{P}^{e}$.

(ii) $K_{\mathfrak{p}}$ is a ramified extension of $F_{\mathfrak{p}}$ and $\zeta$ is a prime in $K_{\mathfrak{p}}-$ the ramified prime case. Now we may assume $\pi=\zeta, p=\pi \pi^{*}$ and $\mathfrak{O}_{\mathrm{p}}$ is generated over $\mathfrak{o}_{\mathfrak{p}}$ by 1 and $\pi$. Here $2 \mathfrak{O}_{\mathfrak{p}}=\mathfrak{P}^{2 e}$. 
(iii) $K_{\mathfrak{p}}$ is a ramified extension of $F_{\mathfrak{p}}$ and $\zeta$ is a unit in $K_{\mathfrak{p}}-$ the ramified unit case. We now have $\zeta^{2}=1-p^{2 h+1} \delta$ for some unit $\delta$ in $o_{p}$ and some rational integer $h$ with $0 \leq h<e$. Put $\pi=(1+\zeta) p^{-h}$ so that $\pi \pi^{*}=p \delta$. Here $\mathfrak{O}_{p}$ consists of the elements $(\alpha+\zeta \beta) p^{-h}$ with $\alpha, \beta \in \mathfrak{o}_{\mathfrak{p}}$ and $\alpha \equiv \beta \bmod p^{h}$; also $2 \mathfrak{O}_{\mathfrak{p}}=\mathfrak{P}^{2 e}$.

In summary, if $K_{\mathfrak{p}} / F_{\mathfrak{p}}$ is a quadratic extension of fields, $\mathfrak{O}_{\mathfrak{p}}$ consists of the elements $(\alpha+\zeta \beta) p^{-h}$ with $\alpha, \beta \in \mathfrak{o}_{\mathfrak{p}}$ and $\alpha \equiv \beta \bmod p^{h}$, where we define $h=0$ in the nondyadic and ramified prime dyadic cases, and $h=e$ in the unramified dyadic case. Note that $T_{\mathfrak{p}}\left(\mathfrak{O}_{\mathfrak{p}}\right)=2 p^{-h_{\mathfrak{o}}}$. Let $\mathfrak{U}_{\mathfrak{p}}$ denote the group of units in $\mathfrak{O}_{\mathfrak{p}}$, and $\mathfrak{u}_{\mathfrak{p}}$ the units in $\mathfrak{o}_{\mathfrak{p}}$. Then $\left[\mathfrak{u}_{\mathfrak{p}}: N_{\mathfrak{p}}\left(\mathfrak{U}_{\mathfrak{p}}\right)\right]=2$ when $\mathfrak{p}$ is ramified dyadic. Also, let $f$ denote the residue class degree of the local field $F_{\mathrm{p}}$.

Lemma 3.2. Assume $\mathfrak{p}$ is ramified dyadic and $c \in \mathfrak{o}_{\mathfrak{p}}$. Then there exists $a \in \mathfrak{D}_{\mathfrak{p}}$ such that $N_{\mathfrak{p}}(a) \equiv c \bmod 2 p^{-h}$.

Proof. Let $a=\alpha+\pi \beta$ where $\alpha, \beta \in \mathfrak{o}_{\mathfrak{p}}$. Then $N_{\mathfrak{p}}(a) \equiv \alpha^{2}+p \delta \beta^{2} \bmod 2 p^{-h}$ (with $\delta=1$ in the ramified prime case) and $\alpha^{2}+p \delta \beta^{2} \equiv c \bmod 2 p^{-h}$ can be solved for $\alpha$ and $\beta$ inductively through successive powers of $p$.

The following result was established as Proposition 2.1 in [J3].

Lemma 3.3. Let $F_{\mathfrak{p}}$ be a dyadic local field with $\mathfrak{p}$ not split in $K$. Then $L_{\mathfrak{p}}^{\prime}=$ $\left\{r \in L_{\mathfrak{p}} \mid p^{h} f(r) \in 2 \mathfrak{o}_{\mathfrak{p}}\right\}$. In particular, $L_{\mathfrak{p}}=L_{\mathfrak{p}}^{\prime}$ when $K_{\mathfrak{p}}$ is an unramified extension of $F_{\mathfrak{p}}$.

Corollary 3.4. $0 \leq m_{\mathfrak{p}}(x) \leq e-h$ for all primitive $x \in L_{\mathfrak{p}}$.

Proof. Let $f(x, z)=1$ where $z \in L_{\mathfrak{p}}$. In the nonsplit dyadic cases take $a \in$ $\mathfrak{P}^{e-h}$. Then $a z \in L_{\mathfrak{p}}^{\prime}$ and consequently $m_{\mathfrak{p}}(x) \leq e-h$.

Since $L_{\mathfrak{p}}$ is a unimodular $\mathfrak{O}_{\mathfrak{p}}$-lattice with rank at least three, it is split by a hyperbolic plane (if $\mathfrak{p}$ splits in $K$ this can be easily verified, otherwise see [Jc, 7.1, 8.1a, 10.3]). Hence $L_{\mathfrak{p}}=H_{\mathfrak{p}} \perp M_{\mathfrak{p}}$ where $H_{\mathfrak{p}}=\mathfrak{O}_{\mathfrak{p}} u+\mathfrak{O}_{\mathfrak{p}} v$ is a hyperbolic plane with $f(u)=f(v)=0$ and $f(u, v)=1$. This choice of $u$ and $v$ will be fixed throughout the local discussion.

Lemma 3.5. Let $F_{\mathfrak{p}}$ be a dyadic local field with $\mathfrak{p}$ ramified in $K$. Then $L_{\mathfrak{p}}=$ $H_{\mathfrak{p}} \perp J_{\mathfrak{p}} \perp B_{\mathfrak{p}}$ where $J_{\mathfrak{p}}$ is a sum of hyperbolic planes and rank $B_{\mathfrak{p}} \leq 2$. If $n$ is odd, $B_{\mathfrak{p}}=\mathfrak{O}_{\mathfrak{p}} w$, with $f(w)$ a unit, and

$$
L_{\mathfrak{p}}^{\prime}=H_{\mathfrak{p}} \perp J_{\mathfrak{p}} \perp \mathfrak{P}^{e-h} w .
$$

If $n$ is even, then $B_{\mathfrak{p}}=\mathfrak{O}_{\mathfrak{p}} z+\mathfrak{O}_{\mathfrak{p}} w$ with $f(z, w)=1$ and $z \in L_{\mathfrak{p}}^{\prime}$, and

$$
L_{\mathfrak{p}}^{\prime}=H_{\mathfrak{p}} \perp J_{\mathfrak{p}} \perp\left(\mathfrak{O}_{\mathfrak{p}} z+\mathfrak{P}^{k} w\right)
$$

where $k=\max \left\{0, e-h-\operatorname{ord}_{p} f(w)\right\}$.

Proof. The splitting of $L_{\mathfrak{p}}$ follows from [Jc, 10.3]. If $B_{\mathfrak{p}}=\mathfrak{O}_{\mathfrak{p}} z+\mathfrak{O}_{\mathfrak{p}} w$ with $f(z, w)=1$ we can arrange that $z \in L_{\mathfrak{p}}^{\prime}$ as follows. Replace $z$ by $z^{\prime}=z+a w$ with $a \in \mathcal{O}_{\mathfrak{p}}$ chosen such that $N_{\mathfrak{p}}(a) \equiv f(z) f(w)^{-1} \bmod 2 p^{-h}$ by Lemma 3.2. Then $p^{h} f\left(z^{\prime}\right) \in 2 \mathfrak{o}_{\mathrm{p}}$. Multiply $z^{\prime}$ by a unit to recover $f\left(z^{\prime}, w\right)=1$. The structure of $L_{\mathfrak{p}}^{\prime}$ follows from Lemma 3.3 . 
We now define the standard isometries needed in the unitary group $U\left(L_{\mathfrak{p}}\right)$. Put $\mu=(1,0)$ when $\mathfrak{p}$ is split. Otherwise, fix $2 \mu=1$ except when $F_{\mathfrak{p}}$ is dyadic and $K_{\mathfrak{p}}$ is either an unramified or a ramified unit extension of $F_{\mathfrak{p}}$; in these exceptional cases fix $2 \mu=1+\zeta \in p^{h} \mathfrak{O}_{\mathfrak{p}}$. Then $T_{\mathfrak{p}}(\mu)=1$. For $s \in M_{\mathfrak{p}}^{\prime}=M_{\mathfrak{p}} \cap L_{\mathfrak{p}}^{\prime}$, define the Eichler transformation $E(u, s)$ by

$$
E(u, s)(x)=x-f(u, x) s+f(s, x) u-\mu f(s) f(u, x) u, \quad x \in L_{p} .
$$

Then $E(u, s) \in S U\left(L_{\mathfrak{p}}\right)$ (note that $\mu f(s) \in \mathfrak{O}_{\mathfrak{p}}$ by Lemma 3.3). Let $\mathscr{E}$ denote the subgroup of $S U(L \mathfrak{p})$ generated by the Eichler transformations $E(u, s)$ and $E(v, s)$ with $s \in M_{\mathfrak{p}}^{\prime}$.

Let $\lambda$ in $\mathfrak{O}_{\mathfrak{p}}$ have $T_{\mathfrak{p}}(\lambda)=0$. The transvection $T_{\lambda}(u)$ is defined by

$$
T_{\lambda}(u)(x)=x+\lambda f(u, x) u, \quad x \in L_{\mathfrak{p}} .
$$

Then $T_{\lambda}(u)$ and $T_{\lambda}(v)$ belong to $S U\left(L_{\mathfrak{p}}\right)$.

Let $\nu \neq 0$ in $\mathfrak{O}_{\mathfrak{p}}$ satisfy $T_{\mathfrak{p}}(\nu)=N_{\mathfrak{p}}(\nu)$. For $r \in L_{\mathfrak{p}}$ with $\nu f(r)^{-1}$ in $\mathfrak{O}_{\mathfrak{p}}$, define the quasi-symmetry $\Psi_{\nu}(r)$ by

$$
\Psi_{\nu}(r)(x)=x-\nu f(r, x) f(r)^{-1} r, \quad x \in L_{p} .
$$

Then $\Psi_{\nu}(r) \in U\left(L_{\mathfrak{p}}\right)$ and $\operatorname{det} \Psi_{\nu}(r)=1-\nu$.

Lemma 3.6. $U\left(H_{\mathfrak{p}}\right)$ is generated by quasi-symmetries and transvections.

Proof. We will reduce $\varphi \in U\left(H_{\mathfrak{p}}\right)$ to the identity using quasi-symmetries and transvections. Let $\varphi(u)=a u+b v$. Since $\Psi_{2}(u-v)$ interchanges $u$ and $v$, assume $a \in \mathfrak{U}_{\mathfrak{p}}$. Put $\lambda=-b a^{-1}$. Then $T_{\mathfrak{p}}(\lambda)=0$, since $f(a u+b v)=0$, and $T_{\lambda}(v) \varphi(u)=a u$. If $T_{\mathfrak{p}}(a) \neq 0$, put $r=a u-v$ and $\nu a=T_{\mathfrak{p}}(a)=-f(r)$ so that $T_{\mathfrak{p}}(\nu)=N_{\mathfrak{p}}(\nu)$. Then $\Psi_{\nu}(r)(a u)=a u-r=v$ and we may assume $\varphi(u)=u$ after applying $\Psi_{2}(u-v)$. If, however, $T_{\mathfrak{p}}(a)=0$, then $T_{a^{*}}(v)(a u)=$ $a u+N_{\mathfrak{p}}(a) v$ with $N_{\mathfrak{p}}(a) \in \mathfrak{u}_{\mathfrak{p}}$. After interchanging $u$ and $v$, it can again be assumed that $\varphi(u)=u$ since $T_{\mathfrak{p}}\left(N_{\mathfrak{p}}(a)\right) \neq 0$. Now $\varphi(v)=c u+v$ with $T_{\mathrm{p}}(c)=0$ and the proof can be completed with the transvection $T_{-c}(u)$.

Proof of Theorem 3.1 (necessity). Condition (i) is clearly necessary and, except in the ramified dyadic situations, (ii) and (iii) are vacuous since $L_{\mathfrak{p}}=L_{\mathfrak{p}}^{\prime}$. Assume, therefore, $\mathfrak{p}$ is ramified dyadic and there exists $\varphi \in S U\left(L_{\mathfrak{p}}\right)$ with $\varphi(x)=y$. Condition (ii) is necessary since $\varphi\left(L_{\mathfrak{p}}^{\prime}\right)=L_{\mathfrak{p}}^{\prime}$. It remains to show that (iii) is satisfied. Let $L_{\mathfrak{p}}=H_{\mathfrak{p}} \perp J_{\mathfrak{p}} \perp B_{\mathfrak{p}}$ as in Lemma 3.5. By Theorem 4.2 in [J3], $U\left(L_{\mathfrak{p}}\right)$ is generated by $\mathscr{E}, U\left(H_{\mathfrak{p}}\right)$ and at most one symmetry in $U\left(B_{\mathfrak{p}}\right)$. Observe first that $\theta(x) \equiv x \bmod \mathfrak{P}^{m} L_{\mathfrak{p}}^{\prime}$ when $\theta$ is an Eichler transformation $E(u, s)$ or $E(v, s)$ with $s \in M_{\mathfrak{p}}^{\prime}$, or when $\theta$ is an element in $U\left(H_{\mathfrak{p}}\right)$ (since $\left.f\left(\theta(x)-x, H_{\mathfrak{p}}\right) \subset \mathfrak{P}^{m}\right)$. Moreover, if $\Psi_{\lambda}(t) \in U\left(H_{\mathfrak{p}}\right)$, then $f(t) \in 2 p^{-h} \mathfrak{o}_{\mathfrak{p}}$ and, consequently, $\lambda \in 2 p^{-h} \mathfrak{O}_{p}$. It remains to study the effect of a quasi-symmetry $\Psi_{\nu}$ from $U\left(B_{\mathfrak{p}}\right)$ in $\varphi$. Since $\operatorname{det} \varphi=1$ and $\operatorname{det} \Psi_{\nu}=1-\nu$, and since $U\left(H_{\mathrm{p}}\right)$ is generated by transvections and quasi-symmetries, there will also have to be quasi-symmetry from $U\left(H_{\mathfrak{p}}\right)$ in $\varphi$; consequently $\nu \in 2 p^{-h} \mathfrak{O}_{\mathfrak{p}}$. When $B_{\mathfrak{p}}=\mathfrak{O}_{\mathfrak{p}} w, f(w)$ is a unit, $\mathfrak{P}^{e-h} w \subset L_{\mathfrak{p}}^{\prime}$ and $\Psi_{\nu}(w)(x) \equiv x \bmod \mathfrak{P}^{m} L_{\mathfrak{p}}^{\prime}$ since $m \leq e-h$. When rank $B_{\mathfrak{p}}=2$, take $B_{\mathfrak{p}}$ as in Lemma 3.5. If $k=0$ there is nothing to prove since $L_{\mathfrak{p}}=L_{\mathfrak{p}}^{\prime}$. Finally assume $k \geq 1$. The quasi-symmetry needed is $\Psi_{\nu}(r)$ with $r=w-f(w) z$ (see [J3, p. 477]). Then $\Psi_{\nu}(r)(x) \equiv x$ $\bmod \mathfrak{P}^{m} L_{\mathfrak{p}}^{\prime}$ since $\mathfrak{P}^{k} r \subset L_{\mathfrak{p}}^{\prime}$. 
Proof of Theorem 3.1 (sufficiency). Assume primitive $x, y$ in $L_{\mathfrak{p}}$ satisfy the three conditions given in Theorem 3.1. Let $x=a u+b v+r$ with $a, b \in$ $\mathfrak{P}^{m}$ and $r \in M_{\mathfrak{p}}$. We prove first there exists $\varphi \in S U\left(L_{\mathfrak{p}}\right)$ such that $\varphi(x)=$ $\pi^{m} u+b^{\prime} v+r^{\prime}$. Note that when the quasi-symmetry $\Psi_{\nu}(t)$ lies in $U\left(H_{\mathfrak{p}}\right)$, with $t \in H_{\mathfrak{p}}$, then $\Psi_{\nu}(t) \Psi_{\nu}(w)^{-1} \in S U\left(L_{\mathfrak{p}}\right)$, where $w$ is as in Lemma 3.5 (since $\left.\operatorname{ord}_{p} f(w) \leq \operatorname{ord}_{p} f(t)\right)$. Assume, therefore, $\operatorname{ord}_{p} a \leq \operatorname{ord}_{p} b$ (otherwise use $\Psi_{2}(u-v)$ to interchange $u$ and $\left.v\right)$. If ord $_{\pi} a>m$ there exists $t_{1} \in M_{\mathfrak{p}}^{\prime}$ such that $\operatorname{ord}_{\pi} f\left(t_{1}, r\right)=m$. The coefficient of $u$ in $E\left(u, t_{1}\right)(x)$ now has order $m$ and we may assume $a=\pi^{m} \varepsilon$ with $\varepsilon \in \mathfrak{U}_{\mathfrak{p}}$. Apply the isometry $u \mapsto \varepsilon^{-1} u$, $v \mapsto \varepsilon^{*} v$ to $x$; although this isometry needed not be in $S U\left(L_{\mathfrak{p}}\right)$, by Lemma 3.6 it can be multiplied by a quasi-symmetry $\Psi_{\nu}(w)$ so that the product is in $S U\left(L_{\mathfrak{p}}\right)$. We may now assume $a=\pi^{m}$. Likewise, assume $y=a u+c v+s$ with $s \in M_{\mathfrak{p}}$. By condition (iii), $x-y=\left(b^{\prime}-c\right) v+\left(r^{\prime}-s\right) \in \mathfrak{P}^{m} L_{\mathfrak{p}}^{\prime}$. Hence $t^{\prime}=a^{-1}\left(r^{\prime}-s\right) \in M_{\mathfrak{p}}^{\prime}$ and $E\left(v, t^{\prime}\right)(x)=a u+c^{\prime} v+s$. Put $\lambda=a^{-1}\left(c-c^{\prime}\right)$. Then $T_{\mathfrak{p}}(\lambda)=0$, since $f(x)=f(y)$, and $T_{\lambda}(v) E\left(v, t^{\prime}\right)(x)=y$, completing the proof.

Corollary 3.7. Assume rank $L_{\mathfrak{p}}=n \geq 3$ and $\mathfrak{p}$ is not ramified dyadic. Then $N\left(L_{\mathfrak{p}}, c\right)=1$ for any $c \in \mathfrak{o}_{\mathfrak{p}}$.

Proof. First $N\left(L_{\mathfrak{p}}, c\right) \leq 1$ by Theorem 3.1 since $L_{\mathfrak{p}}=L_{\mathfrak{p}}^{\prime}$. Also, given $c \in \mathfrak{o}_{\mathfrak{p}}$, there now exists $a \in \mathfrak{O}_{\mathfrak{p}}$ with $T_{\mathfrak{p}}(a)=c$. Put $x=u+a v$. Then $f(x)=c$ and $N\left(L_{\mathfrak{p}}, c\right) \geq 1$.

Proof of Theorem 1.1. Partition $L(c)$ into orbits under $S U(L)$ and let $O(L, c)$ denote the collection of these orbits. Then $N(L, c)=|O(L, c)|$ and there exists a natural mapping

$$
\Gamma: O(L, c) \rightarrow \prod_{\mathfrak{p}} O\left(L_{\mathfrak{p}}, c\right)
$$

into the Cartesian product of the corresponding local orbits $O\left(L_{\mathfrak{p}}, c\right)$. By Corollary 3.7 , the product is essentially over the dyadic primes $\mathfrak{p} \in S$ which ramify in $K$. The map is injective by Theorem 2.1 so it remains to show that $\Gamma$ is surjective. We are given primitive $x_{\mathfrak{p}} \in L_{\mathfrak{p}}$ with $f\left(x_{\mathfrak{p}}\right)=c$ for each dyadic $\mathfrak{p} \in S$ that ramifies. Since $n \geq 3$ there exist similar $x_{\mathfrak{p}} \in L_{\mathfrak{p}}$ for all remaining $\mathfrak{p} \in S$. Also, by hypothesis, there exists primitive $r \in L$ with $f(r)=c$. By Witt's theorem, as in the proof of Theorem 2.1, there exist $\theta_{\mathfrak{p}} \in S U\left(V_{\mathfrak{p}}\right)$ such that $\theta_{\mathfrak{p}}\left(x_{\mathfrak{p}}\right)=r$ for each dyadic $\mathfrak{p} \in S$ that ramifies, and by Theorem 3.1 corresponding $\theta_{\mathfrak{p}}$ in $S U\left(L_{\mathfrak{p}}\right)$ for the remaining $\mathfrak{p} \in S$. There now exists $\psi \in S U(V)$, by strong approximation [S, 5.12], such that $\left\|\psi-\theta_{\mathfrak{p}}^{-1}\right\|_{\mathfrak{p}}$ is small for all ramifying dyadic primes, while $\|\psi\|_{\mathfrak{p}}=1$ at the remaining $\mathfrak{p} \in S$. Put $y=\psi(r)$ so that $f(y)=c$. Then $y$ is close to $x_{\mathfrak{p}}$, and hence primitive in $L_{\mathfrak{p}}$, for all ramifying dyadic $\mathfrak{p}$. For the remaining $\mathfrak{p} \in S, \psi \in S U\left(L_{\mathfrak{p}}\right)$ and hence $y \in L$ is primitive. Moreover, $\psi \theta_{\mathfrak{p}} \in S U\left(L_{\mathfrak{p}}\right)$ and $\psi \theta_{\mathfrak{p}}\left(x_{\mathfrak{p}}\right)=y$ for all ramifying dyadic primes. Hence the orbit of $y$ in $O(L, c)$ is mapped by $\Gamma$ onto the product of the orbits of $x_{\mathfrak{p}}$, for the ramifying dyadic primes.

Remark. Theorem 1.1 is still true if the assumption " $L$ represents $c$ " is replaced by " $V_{\mathrm{q}}$ represents $c$ for all $\mathfrak{q} \notin S$." Then there exists $r \in V$ with $f(r)=c$ by the Hasse Minkowski Theorem. Since $r \in L_{\mathfrak{p}}$ for almost all $\mathfrak{p} \in S$, the above proof is easily modified by including the finite number of exceptions in the approximations $\left\|\psi-\theta_{\mathfrak{p}}^{-1}\right\|_{\mathfrak{p}}$ small. 


\section{LOCAL REPRESENTATIONS: $n$ ODD}

We now compute $N\left(L_{\mathfrak{p}}, c\right)$ when $n=\operatorname{rank} L_{\mathfrak{p}}$ is odd and $\mathfrak{p}$ is ramified dyadic. As in Lemma 3.5,

$$
L_{\mathfrak{p}}=H_{\mathfrak{p}} \perp J_{\mathfrak{p}} \perp \mathfrak{O}_{\mathfrak{p}} w \quad \text { and } \quad L_{\mathfrak{p}}^{\prime}=H_{\mathfrak{p}} \perp J_{\mathfrak{p}} \perp \mathfrak{P}^{e-h} w .
$$

The discriminant $d L_{\mathfrak{p}}=(-1)^{(n-1) / 2} f(w) N_{\mathfrak{p}}\left(\mathfrak{U}_{\mathfrak{p}}\right)$ is an invariant of $L_{\mathfrak{p}}$. The rank and the discriminant determine $L_{\mathfrak{p}}$ when $n$ is odd by [Jc, 10.4]. Thus for each odd rank $n$, there exist two classes of unimodular lattices. Define $\delta\left(L_{\mathfrak{p}}\right)=1$ when $d L_{\mathfrak{p}}=(-1)^{(n-1) / 2} N_{\mathfrak{p}}\left(\mathfrak{U}_{\mathfrak{p}}\right)$, and $\delta\left(L_{\mathfrak{p}}\right)=-1$ otherwise. When $\delta\left(L_{\mathfrak{p}}\right)=1$ we can assume $f(w)=1$.

Lemma 4.1. Assume $n \geq 3$ odd with $\mathfrak{p}$ ramified dyadic. Then

(i) $N\left(L_{\mathfrak{p}}, c\right) \geq 1$ for all $c \in \mathfrak{o}_{\mathfrak{p}}$,

(ii) $N\left(L_{\mathfrak{p}}, c\right)=1$ for all $c \in p \mathfrak{o}_{\mathfrak{p}}$.

Proof. Let $c \in \mathfrak{o}_{\mathfrak{p}}$ and $x=u+b v+a w$ with $a \in \mathfrak{O}_{\mathfrak{p}}$ chosen by Lemma 3.2 such that $N_{\mathfrak{p}}(a) f(w) \equiv c \bmod 2 p^{-h}$. Take $b \in \mathfrak{O}_{\mathfrak{p}}$ such that $f(x)=$ $T_{\mathfrak{p}}(b)+N_{\mathfrak{p}}(a) f(w)=c$. Thus $N\left(L_{\mathfrak{p}}, c\right) \geq 1$. Now assume characteristic $y \in L_{\mathfrak{p}}$ represents $c \in \boldsymbol{p o}_{\mathfrak{p}}$. Then $f\left(y, L_{\mathfrak{p}}^{\prime}\right) \subset \mathfrak{P}$ and $y \equiv a w \bmod \mathfrak{P} L_{\mathfrak{p}}$ for some unit $a \in \mathfrak{U}_{\mathfrak{p}}$. But then $c=f(y) \equiv N_{\mathfrak{p}}(a) f(w) \bmod \mathfrak{P}$ and $c$ must be a unit. It follows that $N\left(L_{\mathfrak{p}}, c\right) \leq 1$, since all noncharacteristic representations are equivalent by Theorem 3.1 .

Lemma 4.2. Assume $n \geq 3$ is odd with $\mathfrak{p}$ ramified prime. Then

$$
N\left(L_{\mathfrak{p}}, c\right)=1+e \text { for all units } c \in \mathfrak{u}_{\mathfrak{p}} .
$$

Proof. Let $x=\zeta^{m}(u+b v)+a w$ with $0 \leq m \leq e, b \in o_{\mathfrak{p}}$ and $a \in \mathfrak{U}_{\mathfrak{p}}$. Then $f\left(x, L_{\mathfrak{p}}^{\prime}\right)=\mathfrak{P}^{m}$ so that $m_{\mathfrak{p}}(x)=m$. Also, $f(x)=? p^{m} b+N_{\mathfrak{p}}(a) f(w)=c$ provided the congruence $N_{\mathfrak{p}}(a) f(w) \equiv c \bmod 2 p^{m}$ can be solved for $a=\alpha+$ $\zeta \beta \in \mathfrak{O}_{\mathfrak{p}}$ Since $m \leq e$, the congruence

$$
N_{\mathfrak{p}}(a) f(w)=\left(\alpha^{2}-p \beta^{2}\right) f(w) \equiv c \quad \bmod 2 p^{m}
$$

can be solved for $\alpha, \beta \in o_{\mathfrak{p}}$ inductively through successive powers of $p$. Hence there exist $1+e$ inequivalent representations of $c$, one for each value of $m$. Moreover, if $y$ is a second representation of $c$ with $m_{\mathfrak{p}}(y)=m$, then $y=$ $\zeta^{m} r+a^{\prime} w$ for some $r \in H_{\mathfrak{p}} \perp J_{\mathfrak{p}}$ and $a^{\prime} \in \mathfrak{U}_{\mathfrak{p}}$. Take $x$ as above. Since $f(r) \in$ $T_{\mathfrak{p}}\left(\mathfrak{O}_{\mathfrak{p}}\right)=2 \mathfrak{o}_{\mathfrak{p}}$, it follows that $N_{\mathfrak{p}}\left(a^{-1} a^{\prime}\right) \equiv 1 \bmod 2 p^{m}$ and, consequently, $a \equiv$ $a^{\prime} \bmod \mathfrak{P}^{m+e}$. Thus $x-y \in \mathfrak{P}^{m} L_{\mathfrak{p}}^{\prime}$ and the two representations are equivalent by Theorem 3.1 . Hence $N\left(L_{\mathfrak{p}}, c\right)=1+e$.

Lemma 4.3. Assume $n \geq 3$ is odd and $\mathfrak{p}$ is ramified unit. Then there exists a characteristic representation of $c \in \mathfrak{u}_{\mathfrak{p}}$ with degree $m, 1 \leq m \leq e-h$, if and only if there exists $a \in \mathfrak{U}_{\mathfrak{p}}$ with $N_{\mathfrak{p}}(a) \equiv c f(w)^{-1} \bmod 2 p^{m-h}$.

Proof. Assume $x=\pi^{m} r+a w$ represent $c$, where $r \in H_{\mathfrak{p}} \perp J_{\mathfrak{p}}$ and $a \in \mathfrak{U}_{\mathfrak{p}}$. Then $c=f(x) \equiv N_{\mathfrak{p}}(a) f(w) \bmod 2 p^{m-h}$. Conversely, if $a \in \mathfrak{U}_{\mathfrak{p}}$ satisfies this congruence, put $x^{\prime}=\pi^{m}(u+b v)+a w$ with $b \in \mathfrak{O}_{\mathfrak{p}}$ chosen such that $f\left(x^{\prime}\right)=c$.

Corollary 4.4. Assume $f=e-h=1$ with $\mathfrak{p}$ ramified unit. Then $L_{\mathfrak{p}}$ characteristically represents $c$ if and only if $c \equiv f(w) \bmod p^{2}$.

Proof. Let $a=\alpha+\pi \beta \in \mathfrak{U}_{\mathfrak{p}}$ so that $N_{\mathfrak{p}}(a)=\alpha^{2}+2 p^{-h} \alpha \beta+p \delta \beta^{2}$. Since $\left|\mathfrak{o}_{\mathfrak{p}} / p\right|=2$, it follows that $\alpha \equiv \delta \equiv 1 \bmod p$ and, by the lemma there is a characteristic representation if and only if $c \equiv f(w) \bmod p^{2}$. 
Lemma 4.5. Assume $n \geq 3$ is odd, $h=e-1$ and $\mathfrak{p}$ is ramified unit. Then $N\left(L_{\mathrm{p}}, c\right)=1$ or 3 .

Proof. By Theorem 3.1 and Lemma 4.3, $N\left(L_{\mathfrak{p}}, c\right)=1$ unless there is an $a=\alpha+\pi \beta \in \mathfrak{U}_{\mathfrak{p}}$ with $N_{\mathfrak{p}}(a)=\alpha^{2}+2 p^{-h} \alpha \beta+p \delta \beta^{2} \equiv c f(w)^{-1} \bmod p^{2}$. Moreover, when such an $a \in \mathfrak{U}_{\mathfrak{p}}$ exists, there is a characteristic representation of $c=f(x)$ with $x=\pi(u+b v)+a w$. Let $a^{\prime}=a+\pi \gamma$ where $\gamma \in \mathfrak{u}_{\mathrm{p}}$ and $\gamma \delta \equiv 2 p^{-e} \alpha \bmod p$. Then $N_{\mathfrak{p}}\left(a^{\prime}\right) \equiv N_{\mathfrak{p}}(a) \bmod p^{2}$ and there exists $b^{\prime} \in \mathfrak{O}_{\mathfrak{p}}$ such that $c=f\left(x^{\prime}\right)$ with $x^{\prime}=\pi\left(u+b^{\prime} v\right)+a^{\prime} w$. Therefore $x-x^{\prime} \notin \mathfrak{P} L_{\mathfrak{p}}^{\prime}$ and $N\left(L_{\mathfrak{p}}, c\right) \geq 3$. Now let $y=\pi r+d w$ be any characteristic representation of $c$. Then $N_{\mathrm{p}}\left(d a^{-1}\right) \equiv 1 \bmod p^{2}$ and it follows that $d \equiv a, a^{\prime} \bmod p$. Hence $y-x \in \mathfrak{P} L_{\mathfrak{p}}^{\prime}$ or $y-x^{\prime} \in \mathfrak{P} L_{\mathfrak{p}}^{\prime}$ so that $N\left(L_{\mathfrak{p}}, c\right) \leq 3$ by Theorem 3.1 .

\section{LOCAL REPRESENTATIONS: $n$ EVEN}

Assume $n=\operatorname{rank} L_{\mathfrak{p}} \geq 4$ is even and $\mathfrak{p}$ is ramified dyadic. Then, as in Lemma 3.5,

$$
L_{\mathfrak{p}}=H_{\mathfrak{p}} \perp J_{\mathfrak{p}} \perp\left(\mathfrak{O}_{\mathfrak{p}} z+\mathfrak{O}_{\mathfrak{p}} w\right) \quad \text { and } \quad L_{\mathfrak{p}}^{\prime}=H_{\mathfrak{p}} \perp J_{\mathfrak{p}} \perp\left(\mathfrak{O}_{\mathfrak{p}} z+\mathfrak{p}^{k} w\right)
$$

where $k=\max \left\{0, e-h-\operatorname{ord}_{p} f(w)\right\}$. There are no characteristic representations when $k=0$ since then $L_{\mathfrak{p}}=L_{\mathfrak{p}}^{\prime}$, that is, $L_{\mathfrak{p}}$ is an even lattice. The discriminant $d L_{\mathfrak{p}}=(-1)^{(n-2) / 2}(f(z) f(w)-1) N_{\mathfrak{p}}\left(\mathfrak{U}_{\mathfrak{p}}\right)$ is an invariant of $L_{\mathfrak{p}}$. Define $\delta\left(L_{\mathfrak{p}}\right)=0$ when $d L_{\mathfrak{p}}=(-1)^{n / 2} N_{\mathfrak{p}}\left(\mathfrak{U}_{\mathfrak{p}}\right)$; in particular, we can assume that $f(z)=0$ in this case. Otherwise, define $\delta\left(L_{\mathfrak{p}}\right)=2$. The invariants $n$, $d L_{\mathfrak{p}}\left(\right.$ or $\left.\delta\left(L_{\mathfrak{p}}\right)\right)$ and $\operatorname{ord}_{p} f(w)$ uniquely determine $L_{\mathfrak{p}}$ by [Jc, 10.4].

Lemma 5.1. Assume $n \geq 4$ is even and $c \in \mathfrak{o}_{\mathfrak{p}}$ with $\mathfrak{p}$ ramified dyadic. Then

(i) $N\left(L_{\mathfrak{p}}, c\right) \leq 1$ when $c$ is a unit,

(ii) $N\left(L_{\mathrm{p}}, c\right)=0$ for all $c \in \mathfrak{o}_{\mathrm{p}}$ with $\operatorname{ord}_{p} c<e-h-k$,

(iii) $N\left(L_{\mathfrak{p}}, c\right) \geq 1$ for all $c \in \mathfrak{o}_{\mathfrak{p}}$ with $\operatorname{ord}_{p} c \geq e-h-k$,

(iv) $N\left(L_{\mathfrak{p}}, c\right)=1$ when $k=0$ for all $c \in \mathfrak{o}_{\mathfrak{p}}$ with $\operatorname{ord}_{p} c \geq e-h$.

Proof. Assume primitive $x \in L_{\mathfrak{p}}$ represents $c \in \mathfrak{o}_{\mathfrak{p}}$. If $x$ is characteristic, then $f\left(x, L_{\mathfrak{p}}^{\prime}\right) \subset \mathfrak{P}$ and $x \equiv a z \bmod \mathfrak{P} L_{\mathfrak{p}}$ for some unit $a \in \mathfrak{U}_{\mathfrak{p}}$. Then $c=f(x) \equiv N_{\mathfrak{p}}(a) f(z) \equiv 0 \bmod \mathfrak{P}$, since $f(z) \in 2 p^{-h} \mathfrak{o}_{\mathfrak{p}}$ by Lemma 3.3. This proves (i) since there is at most one noncharacteristic representation (up to equivalence). Since $\operatorname{ord}_{p} f(w) \geq e-h-k$, the lattice $L_{\mathrm{p}}$ cannot represent any element $c$ with $\operatorname{ord}_{p} c<e-h-k$; this proves (ii). As in Lemma 4.1, there is a noncharacteristic representation of each $c \in \mathfrak{o}_{\mathrm{p}}$ with $\operatorname{ord}_{p} c \geq e-h-k$. When $k=0$ this is the only representation (up to equivalence).

Lemma 5.2. Assume $n \geq 4$ is even, $e=1, k>0$ and $\mathfrak{p}$ ramified prime. Then $N\left(L_{\mathfrak{p}}, c\right)=2^{f}$ for $c \in 2 \mathfrak{o}_{\mathfrak{p}}$.

Proof. Since $k>0$ and $e=1$ it follows that $k=1$ and $f(w)$ is a unit. Also $f(z) \in 2 \mathfrak{o}_{\mathfrak{p}}$ by Lemma 3.3. Put $x=\zeta(u+b v)+d z+a \zeta w$ with $a, b \in \mathfrak{o}_{\mathfrak{p}}$ and $d \in \mathfrak{u}_{\mathfrak{p}}$. Then $f(x)=2 p b+d^{2} f(z)+p a^{2} f(w)$. For fixed $d$, choose $a \in \mathfrak{o}_{\mathfrak{p}}$ such that $p a^{2} f(w) \equiv c-d^{2} f(z) \bmod 4$, and then $b$ such that $f(x)=c$. Varying $d$, this gives $2^{f}-1$ inequivalent characteristic representations of $c$. Hence $N\left(L_{\mathfrak{p}}, c\right) \geq 2^{f}$, since there also exists a noncharacteristic representation of $c$ 
from Lemma 5.1. Let $y=\zeta r+d^{\prime} z+a^{\prime} \zeta w$ be a characteristic representation of $c$, where $r$ lies in $H_{\mathfrak{p}} \perp J_{\mathfrak{p}}$. Then $d \equiv d^{\prime} \bmod \mathfrak{P}$ for one of the $x$ 's above and $y-x \equiv\left(a^{\prime}-a\right) \zeta w \bmod \mathfrak{P} L_{\mathfrak{p}}^{\prime}$. Since $f(y)=c=f(x)$, it follows that $N_{\mathfrak{p}}\left(a^{\prime}\right) \equiv a^{2} \bmod 2$ and hence $a^{\prime} \equiv a \bmod \mathfrak{P}$. Thus $x$ and $y$ are equivalent representations, and $N\left(L_{\mathfrak{p}}, c\right) \leq 2^{f}$.

Lemma 5.3. Assume $n \geq 4$ is even, with $\mathfrak{p}$ ramified unit, $h=e-1, k>0$ and $\delta\left(L_{\mathrm{p}}\right)=0$. Then

(i) $N\left(L_{\mathfrak{p}}, c\right)=2^{f+1}-1$ when $\operatorname{ord}_{p} c \geq 2$,

(ii) $N\left(L_{\mathfrak{p}}, c\right)=2^{f}-1$ when $\operatorname{ord}_{p} c=1$.

Proof. Since $k>0$ and $h=e-1$, it follows that $k=1$ and $f(w)$ is a unit. Since $d L_{\mathfrak{p}}=(-1)^{n / 2} N_{\mathfrak{p}}\left(\mathfrak{U}_{\mathfrak{p}}\right)$ and ord $\operatorname{ord}_{p} f(w)$ determine $L_{\mathfrak{p}}$, we may assume $f(z)=0$ and $f(w)=1$. Put $x=\pi(u+b v)+d z+a \pi w$ with $a \in \mathfrak{o}_{\mathfrak{p}}, b \in \mathfrak{O}_{\mathfrak{p}}$ and $d \in \mathfrak{u}_{\mathfrak{p}}$. Then $f(x)=p \delta T_{\mathfrak{p}}(b)+a d 2 p^{-h}+p \delta a^{2}$.

Assume first $\operatorname{ord}_{p} c \geq 2$. Fix $d \in \mathfrak{u}_{\mathfrak{p}}$ and put $a=0$ or $a=-2 p^{-e} d \delta^{-1} \in \mathfrak{u}_{\mathfrak{p}}$, and choose $b \in \mathfrak{O}_{\mathfrak{p}}$ such that $f(x)=c$. Since there are $2^{f}-1$ choices for $d \bmod p$, this gives $2\left(2^{f}-1\right)$ inequivalent characteristic representations of $c$, and hence $N\left(L_{\mathfrak{p}}, c\right) \geq 2^{f+1}-1$. Conversely, if $y=\pi r+d^{\prime} z+a^{\prime} \pi w$ represents $c$, then $d^{\prime} \equiv d \bmod \mathfrak{P}$ for one of the $x$ 's constructed above. It follows that $a^{\prime} \equiv 0 \bmod \mathfrak{P}$ or $a^{\prime} \equiv 2 p^{-e} d \delta^{-1} \bmod \mathfrak{P}$, and hence $y$ is equivalent to one of these $x$ 's. Thus $N\left(L_{\mathfrak{p}}, c\right)=2^{f+1}-1$.

Now assume $p^{-1} c$ is a unit. Put $b=0$ and choose $a \in u_{p}$ such that $a^{2} \delta \not \equiv p^{-1} c \bmod P$. Then choose $d \in u_{p}$ so that $f(x)=c$. Since there are $2^{f}-2$ choices for $a$ mod $p$, this gives $2^{f}-2$ inequivalent characteristic representations of $c$. Hence $N\left(L_{p}, c\right) \geq 2^{f}-1$. Conversely, any characteristic representation of $c$ is equivalent to one of those just constructed, so that $N\left(L_{p}, c\right)=2^{f}-1$.

Lemma 5.4. Assume $n \geq 4$ is even, with $\mathfrak{p}$ ramified unit, $h=e-1, k>0$ and $\delta\left(L_{\mathfrak{p}}\right)=2$. Then

(i) $N\left(L_{\mathfrak{p}}, c\right)=1$ when $\operatorname{ord}_{p} c \geq 2$,

(ii) $N\left(L_{\mathfrak{p}}, c\right)=2^{f}+1$ when $\operatorname{ord}_{p} c=1$.

Proof. Since $\delta\left(L_{\mathfrak{p}}\right)=2$ and $k=1, V_{\mathfrak{p}}$ cannot be hyperbolic so that $f(w)$ is a unit and the binary lattice $\mathfrak{O}_{\mathfrak{p}} z+\mathfrak{O}_{\mathfrak{p}} w$ must be anisotropic. Hence

$$
\operatorname{ord}_{p} f(d z+\pi a w)=1
$$

for all $d \in \mathfrak{U}_{\mathfrak{p}}$ and $a \in \mathfrak{D}_{\mathfrak{p}}$. Therefore, $L_{\mathfrak{p}}$ cannot characteristically represent any $c \in p^{2} \mathfrak{o}_{\mathfrak{p}}$, proving (i). Now assume $\operatorname{ord}_{p} c=1$. Fix $a \in \mathfrak{o}_{\mathfrak{p}}$. Since $f(z+\pi a w)=p \varepsilon$ for some unit $\varepsilon$, there exist $d \in \mathfrak{u}_{\mathfrak{p}}$ and $b \in \mathfrak{O}_{\mathfrak{p}}$ such that $x=\pi(u+b v)+d(z+\pi a w)$ represents $c$. Hence there exist $2^{f}$ inequivalent characteristic representations of $c$ and $N\left(L_{\mathrm{p}}, c\right) \geq 2^{f}+1$. Conversely, since $a$ mod $\mathfrak{P}$ uniquely determines $d$ mod $\mathfrak{P}$, any characteristic representation of $c$ is equivalent to one constructed above. Hence $N\left(L_{p}, c\right)=2^{f}+1$.

\section{QuADRATIC EXTENSIONS OF $\mathbf{Q}$}

Let $F=\mathbf{Q}, K=\mathbf{Q}(\sqrt{m})$, with $m$ a square free integer, and $\mathfrak{o}_{F}=\mathbf{Z}$. Let $p$ be a rational prime. Then $p$ splits in $K$ if either $p=2$ and $m \equiv 1 \bmod 8$, 
or $p$ is odd and $\left(\frac{m}{p}\right)=1$. Otherwise, for $p=2$, we have an unramified extension if $m \equiv 5 \bmod 8$, a ramified unit extension with $h=0$ if $m \equiv 3$ $\bmod 4$, and a ramified prime extension if $m$ is even. Since $-1 \notin N_{\mathfrak{p}}\left(\mathfrak{U}_{\mathfrak{p}}\right)$ at the dyadic prime when $m \equiv 3 \bmod 4$, we may assume $f(w)=\delta\left(L_{\mathfrak{p}}\right)$ in $L_{\mathfrak{p}}$ when $n$ is odd, and $f(z)=\delta\left(L_{\mathfrak{p}}\right)$ and $f(w)=1$ when $n$ is even and $L_{\mathfrak{p}}$ is not even, in this situation.

Theorem 6.1. Let $L$ be a unimodular lattice on an indefinite hermitian space $V$ of dimension $n \geq 3$ over $K=\mathbf{Q}(\sqrt{m})$. Let $c \neq 0$ be a rational integer represented by $L$, and assume the local Witt index of $V \perp\langle-c\rangle$ at the real prime is at least two when $m<0$. Then

(i) $N(L, c)=3$ when $m \equiv 3 \bmod 4, c \equiv \delta\left(L_{\mathfrak{p}}\right) \bmod 4$ and $L_{\mathfrak{p}}$ is not even,

(ii) $N(L, c)=2$ when $m \equiv 2 \bmod 4, n \equiv c \bmod 2$ and $L_{\mathfrak{p}}$ is not even,

(iii) $N(L, c)=1$ otherwise.

Proof. This follows from Theorem 1.1 and the local results in the two previous sections since $e=f=1$ for any ramifying dyadic prime.

Remark. This theorem also provides information about the orbits in $L(c)$ under the action of the group $U(L)$. Clearly, when $N(L, c)=1$ there is also only one orbit under the action of $U(L)$. However, when $N(L, c)>1$ there will also be at least two orbits under $U(L)$, for no locally characteristic vector in $L(c)$ can be mapped by $U(L)$ into a locally noncharacteristic vector.

Example. Assume $m \equiv 3 \bmod 4$ and $L$ is a free $\mathfrak{O}_{K}$-module with orthogonal basis $x_{1}, \ldots, x_{n}$, where $f\left(x_{i}\right)=-1$ for $1 \leq i \leq r$ and $f\left(x_{i}\right)=1$ for $r+1 \leq i \leq n$. Then $d L_{2}=(-1)^{r} \in N_{2}\left(\mathfrak{U}_{2}\right)$ if and only if $r$ is even (and then $\delta\left(L_{2}\right)=0$ or 1$)$. Assume $1 \leq r<n$ where $m<0$ so that $L$ is indefinite. Let $c \in \mathbf{Z}$ be nonzero. When $m<0$, assume $r \geq 2$ if $c<0$, and $r \leq n-2$ if $c>0$, so that the index condition on $V \perp\langle-c\rangle$ is satisfied. Then $N(L, c)=3$ if either $n$ is even and $c \equiv 2 r \bmod 4$, or $n$ is odd and $c \equiv 2 r+1 \bmod 4$; otherwise $N(L, c)=1$.

\section{Cyclotomics Fields}

We now consider hermitian forms over the cyclotomic field $K=\mathbf{Q}(\omega)$, with $\omega$ a primitive $m$ th root of unity, and $F=\mathbf{Q}(\omega) \cap \mathbf{R}=\mathbf{Q}\left(\omega+\omega^{*}\right)$ the maximal real subfield of $K$. The involution $*$ on $K$ is complex conjugation. Let $\mathfrak{o}=\mathfrak{o}_{F}$ be the ring of algebraic integers in $F$.

Theorem 7.1. Let $L$ be a unimodular lattice on an hermitian space $V$ of dimension $n \geq 3$ over $K=\mathbf{Q}(\omega)$ where $\omega$ is a primitive mth root of unity and $m \geq 3$ is odd. Assume $L$ represents $c \in o_{F}, c \neq 0$, and the local Witt index of $V \perp\langle-c\rangle$ is at least two for some archimedean prime spot. Then $N(L, c)=1$.

Proof. When $m \geq 3$ is odd, the prime 2 is unramified in $K$ and, consequently, $N(L, c) \leq 1$ for any nonzero $c \in \mathfrak{o}_{F}$, by Theorem 1.1 .

When $m=2^{k} \geq 4$, it follows that $l \in K$ where $l^{2}=-1$, and hence $K=F(l)$. Moreover, 2 totally ramifies in $K$. When $\mathfrak{p} \in S$ is the unique 
dyadic prime spot, $K_{\mathfrak{p}} / F_{\mathfrak{p}}$ is a dyadic ramified unit extension with $e=\operatorname{ord}_{2} p=$ $\frac{1}{2} \phi\left(2^{k}\right)=2^{k-2}$. Since $l^{2}=1-p^{e} \varepsilon$ with $\varepsilon$ a unit in $\mathfrak{o}_{\mathfrak{p}}$, it follows that $h=0$ when $e=1$, and $e>h \geq \frac{1}{2}(e-1)$, in general. Therefore, $e=2$ and $h=1$ when $m=8$.

Lemma 7.2. Let $K=\mathbf{Q}(\omega)$ where $\omega$ is a primitive mth root of unity with $m=2^{k} \geq 4$. If $\mathfrak{p}$ is the unique prime spot over 2 , then $K_{\mathfrak{p}} / F_{\mathfrak{p}}$ is a ramified unit extension with $e=m / 4$ and $h=e-1$.

Proof. It remains to show $h=e-1$ when $m \geq 16$. Put $p=\omega+\omega^{*} \in \mathfrak{o}_{\mathfrak{p}}$. Since $e$ is a 2 -power, $\left(\begin{array}{c}e \\ e / 2\end{array}\right) \equiv 2 \bmod 4$, and $\left(\begin{array}{l}e \\ i\end{array}\right) \equiv 0 \bmod 4$ for $1 \leq i<e / 2$. Hence $\omega^{e}=l$ and $p^{e}=\left(\omega+\omega^{*}\right)^{e} \equiv 2 \bmod 4$, so that $p$ is prime in $F_{\mathrm{p}}$. Put

$$
a=1+p^{e / 2}+p^{3 e / 4}+p^{7 e / 8}+\cdots+p^{(e-1) e / e} .
$$

Then $a \in \mathfrak{o}_{\mathfrak{p}}$ and $(a l)^{2} \equiv 1-p^{2 e-1} \bmod 4$. Since $K_{\mathfrak{p}}=F_{\mathfrak{p}}(a l)$ it follows that $h=e-1$.

Now return to the general cyclotomic field $K=\mathbf{Q}(\omega)$ where $m=2^{k} m^{\prime}$ with $k \geq 2$ and $m^{\prime}$ odd. Let $\mathfrak{p}$ be a dyadic prime spot in $S$. Denote by $g$ the number of dyadic spots in $S$. Each $\mathfrak{p}$ will now ramify in $K$. As before, let $e=\operatorname{ord}_{p} 2$ be the ramification index in $F_{\mathrm{p}}$ and hence also in $F$. Then $e=2^{k-2}$, since 2 is unramified in $\mathbf{Q}\left(\omega^{\prime}\right)$ where $\omega^{\prime}$ is a primitive $m^{\prime}$ th root of unity. Let $f$ denote the residue class degree of $p$ in $F$ (and hence also in $K=F(l)$ ). Then $f \geq 1$ is minimal such that $2^{f} \equiv 1 \bmod m^{\prime}$ (with $f=1$ when $\left.m^{\prime}=1\right)$ and $2 e f g=\phi(m)$. Hence $f g=\phi\left(m^{\prime}\right)$. Define $g^{\prime}=g^{\prime}(L)$ $(\leq g)$ to be the number of dyadic primes $\mathfrak{p}$ where $\delta\left(L_{\mathfrak{p}}\right)=1$ when $n$ is odd, and where $\delta\left(L_{\mathfrak{p}}\right)=0$ and $L_{\mathfrak{p}}$ is not an even lattice when $n$ is even. Define $g^{\prime \prime}\left(\leq g-g^{\prime}\right)$ to be the number of dyadic primes where $\delta\left(L_{\mathrm{p}}\right)=2$ and $L_{\mathrm{p}}$ is not even.

Theorem 7.3. Let $L$ be a unimodular lattice on an hermitian space $V$ of dimension $n \geq 3$ over $K=\mathbf{Q}(\omega)$, where $\omega$ is a primitive mth root of unity. Assume $L$ represents the nonzero integer $c \in \mathbf{Z}$ and that the local Witt index of $V \perp\langle-c\rangle$ is at least two at some archimedean prime spot. Then, when $m \equiv 0$ $\bmod 8$,

(i) $N(L, c)=\left(2^{f+1}-1\right)^{g^{\prime}}$ when $n \equiv c \equiv 0 \bmod 2$,

(ii) $N(L, c)=3^{g^{\prime}}$ when $n \equiv c \equiv 1 \bmod 2$,

(iii) $N(L, c)=1$ otherwise;

and when $m \equiv 4 \bmod 8$,

(iv) $N(L, c)=\left(2^{f+1}-1\right)^{g^{\prime}}$ when $2 n \equiv c \equiv 0 \bmod 4$,

(v) $N(L, c)=\left(2^{f}-1\right)^{g^{\prime}}\left(2^{f}+1\right)^{g^{\prime \prime}}$ when $n \equiv 0 \bmod 2$ and $c \equiv 2 \bmod 4$,

(vi) $N(L, c)=3^{g^{\prime}}$ when $n \equiv c \equiv 1 \bmod 2$, provided $f$ is even when $c \equiv 3$ $\bmod 4$

(vii) $N(L, c)=3^{g-g^{\prime}}$ where $n$ and $f$ are odd, and $c \equiv 3 \bmod 4$,

(viii) $N(L, c)=1$ otherwise.

Proof. Apply Theorem 1.1 and the local results in $\S \S 4$ and 5. The condition $m \equiv 0$ mod 8 ensures that locally $e \geq 2$ with $e-h=1$ for any dyadic prime, so that $c \equiv 0,1 \bmod p^{2}$. However, $e=1$ and $p=2$ when $m \equiv 4 \bmod 8$. Parts (i), (iv) and (v) then follow from Lemmas 5.3 and 5.4. 
Now assume $n$ is odd. As in Lemma 7.2 , take $p^{e} \equiv 2 \bmod 4$ and $\zeta^{2} \equiv$ $1-p^{2 e-1} \bmod 4$. Consider first $\delta\left(L_{\mathfrak{p}}\right)=-1$. Choose $b \in \mathfrak{u}_{\mathfrak{p}}$ such that $X^{2}+$ $X \equiv b \bmod p$ has no roots; in particular, take $b=1$ when $f$ is odd. Put $f(w)=1+p b \notin N_{\mathfrak{p}}\left(\mathfrak{U}_{\mathfrak{p}}\right)$. When $e \geq 2$, the congruence in Lemma 4.3 has no solutions for $c$ odd, and hence there are no characteristic representations of $c$. When $e=1$, so that $p=2$ and $\pi=1+l$, the congruence has a solution if and only if $c \equiv 3 \bmod 4$ and $f$ is odd (that is when $b=1$ ). Finally let $\delta\left(L_{\mathfrak{p}}\right)=1=f(w)$. If $c$ is odd, the congruence in Lemma 4.3 can always be solved when $e \geq 2$, or when $c \equiv 1 \bmod 4$. However, if $e=1$ and $c \equiv 3$ mod 4 , the congruence can only be solved when $f$ is even, since it reduces to solving $X^{2}+X \equiv 1 \bmod 2$. The remaining parts of the theorem now follow from Lemma 4.5 .

Remarks. The value of $N(L, c)$ can be computed when $c \notin \mathbf{Z}$, but it now also depends on the varying values of $\operatorname{ord}_{p} c$ at the dyadic primes. The comment following Theorem 6.1 also applies here.

\section{ACKNOWLEDGMENT}

The author thanks the referee for suggesting a number of improvements in the presentation.

\section{REFERENCES}

[G] L. J. Gerstein, Integral decomposition of hermitian forms, Amer. J. Math. 92 (1970), 398418.

[Jc] R. Jacobowitz, Hermitian forms over local fields, Amer. J. Math. 84 (1962), 441-465.

[J1] D. G. James, On Witt's theorem for unimodular quadratic forms, Pacific J. Math. 26 (1968), 303-316 and 33 (1970), 645-652.

[J2] _ Representations by integral quadratic forms, J. Number Theory 4 (1972), 321-329.

[J3] _ Invariant submodules of unimodular hermitian forms, Pacific J. Math. 72 (1977), 471-482.

[J4] _ _ Integral sums of squares in algebraic number fields, Amer. J. Math. 113 (1991), 129146.

[K] D. Z. Kilhefner, Integral extensions of isometries of unimodular hermitian forms, Ph. D. Thesis, Pennsylvania State Univ., 1971.

[N] V. V. Nikulin, Integral symmetric bilinear forms and some of their applications, Math. USSR-Izv. 14 (1980), 103-167.

[O'M] O. T. O'Meara, Introduction to quadratic forms, Springer-Verlag, New York, 1963.

[S] G. Shimura, Arithmetic of unitary groups, Ann. of Math. 79 (1964), 369-409.

[W] C. T. C. Wall, On the orthogonal groups of unimodular quadratic forms, Math. Ann. 147 (1962), 328-338.

Department of Mathematics, Pennsylvania State University, University Park, PennSYLVANIA 16802

E-mail address: james@math.psu.edu 